\title{
Relação peso-comprimento e fator de condição de Oligosarcus hepsetus (Cuvier, 1829) no Parque Estadual da Serra do Mar - Núcleo Santa Virgínia, Mata Atlântica, estado de São Paulo, Brasil
}

\author{
Leandro Muller Gomiero ${ }^{1,2}$, Gilberto Aparecido Villares Junior ${ }^{1}$ \& Francisco Manoel de Souza Braga $^{1}$ \\ ${ }^{1}$ Departamento de Zoologia, Instituto de Biociências, Universidade Estadual Paulista - UNESP, \\ Av. 24-A, n. 1515, CP. 199, CEP 13506-900, Rio Claro, SP, Brasil \\ ${ }^{2}$ Autor para correspondência: Leandro Muller Gomiero, e-mail: leanmg@rc.unesp.br
}

GOMIERO, L.M., VILLARES JUNIOR, G.A. BRAGA, F.M.S. Length-weight relationship and condition factor for Oligosarcus hepsetus (Cuvier, 1829) in Serra do Mar State Park - Santa Virgínia Unit, Atlantic Forest, São Paulo, Brazil. Biota Neotrop. 10(1): http://www.biotaneotropica.org.br/v10n1/en/ abstract?article+bn01610012010.

\begin{abstract}
In this work the length-weight relationship and the condition factor of Oligosarcus hepsetus were analyzed in the Grande river within the Santa Virgínia Unit of the Serra do Mar State Park, State of São Paulo. The length-weight relationship was esteemed for immature, females and male, through the expression $\mathrm{W}=\mathrm{aL}^{\mathrm{b}}$. The parameters $a$ and $b$ were dear after logarithmic transformation of the data of weight and length and subsequent adjustment of a straight line to the points for the method of the minimum square. For females and males were obtained the equations of the length-weight relationships by period of the year (autumn, winter, spring and summer), using the total weight and the weight without the gonads. The factor of condition of the immature was higher than for males and females, possibly, due to the energy expense with the development of the oocytes and in smaller degree of the spermatozoids. This species presented larger values of condition factor in the autumn due to the fat accumulation and in the other stations these values decreased due to the long reproductive period. The environment conditions of the Atlantic forest seem to be primordial for the development of the reproductive strategies presented by Oligosarcus hepsetus.
\end{abstract}

Keywords: length-weight relationship, condition factor, Oligosarcus hepsetus, Atlantic Forest.

GOMIERO, L.M., VILLARES JUNIOR, G.A. BRAGA, F.M.S. Relação peso-comprimento e fator de condição de Oligosarcus hepsetus (Cuvier, 1829) no Parque Estadual da Serra do Mar - Núcleo Santa Virgínia, Mata Atlântica, estado de São Paulo, Brasil. Biota Neotrop. 10(1): http://www.biotaneotropica.org.br/v10n1/pt/ abstract?article+bn01610012010.

Resumo: Foram analisados a relação peso-comprimento e o fator de condição de Oligosarcus hepsetus no Rio Grande do Parque Estadual da Serra do Mar - Núcleo Santa Virgínia, São Paulo. A relação peso-comprimento foi estimada para imaturos, fêmeas e machos, através da expressão $\mathrm{P}=\mathrm{aC}$. Os parâmetros a e $\mathrm{b}$ foram estimados após transformação logarítmica dos dados de peso e comprimento e subsequente ajuste de uma linha reta aos pontos pelo método dos mínimos quadrados. Para fêmeas e machos foram obtidas as equações da relação pesocomprimento por período do ano (outono, inverno, primavera e verão), utilizando o peso total e o peso sem as gônadas. O fator de condição dos imaturos foi mais alto do que para machos e fêmeas, possivelmente, devido ao gasto energético com o desenvolvimento dos ovócitos e em menor grau dos espermatozóides. Esta espécie apresentou maiores valores de fator de condição no outono devido ao acúmulo de gordura e nas demais estações estes valores diminuíram devido ao longo período reprodutivo. As condições ambientais da Mata Atlântica parecem ser primordiais para o desenvolvimento das estratégias reprodutivas apresentadas por Oligosarcus hepsetus.

Palavras-chave: relação peso-comprimento, fator de condição, Oligosarcus hepsetus, Mata Atlântica. 


\section{Introdução}

A Mata Atlântica vem sofrendo continuamente a ação antrópica sob a forma de devastação florestal, exploração dos recursos naturais, poluição, ocupação humana desordenada, fragmentação, etc. $\mathrm{O}$ seu status de conservação é definido como crítico (Araújo-Lima et al. 2004), devido aos riscos para toda fauna e flora desse importante bioma do Brasil.

A ictiofauna é igualmente afetada das mais variadas maneiras, como, por exemplo, pela degradação da qualidade da água ou do hábitat ou pela combinação dos dois fatores, tendo como consequências elevadas cargas de material em suspensão e o resultante assoreamento (Araújo 1998), ocorrendo tanto em córregos de montanhas como em Rios de planície.

O Rio Paraíba do Sul, apesar de sua enorme importância como principal bacia hidrográfica entre São Paulo e o Rio de Janeiro, tem recebido pouca atenção do ponto de vista de estudos dos ecossistemas. Sua bacia hidrográfica apresenta área de cerca de $57.000 \mathrm{~km}^{2}$, localizando-se em fundo de vale, entre as serras do Mar e da Mantiqueira (Araújo 1998). Em áreas mais elevadas, naturalmente protegidas pela altitude, as regiões de cabeceiras abrigam diversidade baixa e alto endemismo de espécies de peixes. Devido a isto, estas regiões são biologicamente importantes e estudos nessas áreas devem ser prioritários (Castro \& Menezes 2001, Menezes 2003, BeneditoCecilio et al. 2004).

Os peixes com hábitos alimentares carnívoros aumentam a estabilidade do ecossistema aquático, visto que atuam no ajuste da abundância de espécies de presas (Nikolskii 1963, Popova 1978) e suas presenças denotam uma relativa estabilidade do ambiente. O peixe denominado cachorro-magro, Oligosarcus hepsetus (Cuvier 1829), é um carnívoro de pequeno a médio porte que habita principalmente lugares rasos com vegetação densa em tributários ou em grandes rios (Araújo et al. 2005). Esta espécie está distribuída em lagoas, reservatórios e rios na região costeira do Sudeste-Sul do Brasil entre Santa Catarina e o Rio de Janeiro (Menezes 1987).

A relação peso-comprimento é uma maneira fácil e rápida de descrever o crescimento, sem levar em conta a idade do peixe. Tem sido usada para converter comprimento em peso, conhecendo-se o comprimento, ou vice e versa (Nomura 1962), como sendo uma das etapas para o estudo do fator de condição (Braga 1986, 1993, 1997), usada como um parâmetro no estudo da biomassa para estimativas de populações naturais ou em cativeiro (Ricker 1975, Santos 1978), ou na estimativa do stand crop em estudos bio-ecológicos (Lotrich 1973).

Vazzoler (1996) comenta que o fator de condição é um importante indicador do grau de higidez de um indivíduo e seu valor reflete as condições nutricionais recentes e/ou gastos das reservas em atividades cíclicas, sendo possível relacioná-lo às condições ambientais e aos aspectos comportamentais das espécies. Este parâmetro é uma ferramenta importante e eficiente para evidenciar mudanças na condição dos peixes ao longo do ano, podendo ser usado para indicar o período reprodutivo, períodos de alterações alimentares e de acúmulo de gordura (Gomiero \& Braga 2003, 2005, 2006), assim como mudanças sazonais nas condições do ambiente (Braga et al. 1985). Porém, na floresta ombrófila densa, típica da área de estudo as condições climáticas são pouco variáveis e praticamente não há estação seca (Tonhasca Jr. 2005), tornando o ambiente estável quanto a esses aspectos.

O objetivo do presente trabalho é caracterizar a relação pesocomprimento e o fator de condição de Oligosarcus hepsetus, peixe carnívoro típico dos rios da Mata Atlântica do estado de São Paulo, relacionando-os com a sazonalidade na reprodução e na alimentação ao longo do ano, tanto quanto ao sexo como também em relação aos indivíduos jovens e adultos.

\section{Material e Métodos}

O local de coleta foi o Rio Grande (bacia do Rio Paraíba do Sul) no Núcleo Santa Virgínia-Parque Estadual da Serra do Mar. Esta área protegida cobre 16.000 ha e se insere nos municípios de São Luiz do Paraitinga, Natividade da Serra, Cunha e Ubatuba, localizada entre as coordenadas $23^{\circ} 24^{\prime}-23^{\circ} 17^{\prime} \mathrm{S}$ e $45^{\circ} 03^{\prime} \mathrm{O}$.

Foram efetuadas 12 amostragens mensais de janeiro a dezembro de 2004. Em cada local foram utilizadas redes com malhas de 1,5; 2,$0 ; 2,5 ; 3,0 ; 3,5$ e 4 cm, medidas entre nós adjacentes. Cada conjunto de malhagem específica possuía $10 \mathrm{~m}$ de comprimento e $1,5 \mathrm{~m}$ de altura, totalizando $60 \mathrm{~m}$. Além destas redes, foram utilizados covos e peneiras para a captura dos menores exemplares. O esforço de pesca foi padronizado mantendo-se constante o tempo e o número dos apetrechos de pesca utilizados no local de amostragem em cada período de coleta.

Para cada exemplar foram tomados o peso total (g), o comprimento total $(\mathrm{cm})$ e o sexo. Os imaturos foram determinados macroscopicamente pela visualização das gônadas, considerando coloração, transparência e vascularização superficial. Para a determinação de machos e de fêmeas procedeu-se à visualização das gônadas quanto a coloração, forma e presença do líquido espermático ou dos ovócitos, respectivamente.

As frequências numéricas nas classes de comprimento total de machos e de fêmeas foram analisadas com o teste KolmogorovSmirnov (Vanzolini 1993), $\alpha=0,05$, e as proporções mensais e sazonais entre machos e fêmeas de Oligosarcus hepsetus foram analisadas com Tabelas de contingência do tipo i x 2 (sendo i > 2) (Vanzolini 1993), $\alpha=0,05$.

$\mathrm{O}$ fator de condição foi estimado utilizando inicialmente a relação peso-comprimento $\left(\mathrm{P}=\mathrm{aC}^{\mathrm{b}}\right)$, após transformação logarítmica dos dados, obtendo-se lny $=\ln \mathrm{A}+\mathrm{B} \ln \mathrm{x}$, que pelo método dos mínimos quadrados obtêm-se A e B de onde obtêm-se a = e $\mathrm{e}^{\mathrm{A}}, \mathrm{b}=\mathrm{B}$ (Santos 1978, Braga 1986). O fator de condição $\left(K=\mathrm{P} / \mathrm{C}^{\mathrm{b}}\right)$ foi estimado para fêmeas, machos e imaturos (Le-Cren 1951, Weatherley 1972), fazendo-se para cada grupo a relação peso-comprimento e assim utilizando-se a constante $\mathrm{b}$ estimada, respectivamente. $\mathrm{O}$ fator de condição obtido dessa maneira foi relacionado com os comprimentos individuais respectivos de cada grupo. Para verificar a correspondência do fator de condição para os grupos de fêmeas, machos e imaturos, foi aplicada a prova não-paramétrica de Kruskal-Wallis seguido de um teste "a posteriori” de Tukey (Zar 1996).

O fator de condição também foi analisado sazonalmente, para fêmeas e para machos, considerando para o seu cálculo o peso total do peixe $\left(\mathrm{K}_{1}\right)$ e o peso total menos o peso da gônada $\left(\mathrm{K}_{2}\right)$ (Braga \& Gennari Filho 1990), para cada valor de K sazonal foi estimado o intervalo de confiança $(\alpha=0,05)$.

\section{Resultados}

Nas doze coletas de 2004 foram capturados e analisados 117 exemplares de Oligosarcus hepsetus, sendo 21 exemplares imaturos, 44 fêmeas e 52 machos. Os exemplares de Oligosarcus hepsetus alcançaram comprimentos totais de 4,2 a $22 \mathrm{~cm}$. O peso total capturado foi de $3.416,98 \mathrm{~g}$, sendo que as fêmeas contribuíram com $1.830,22 \mathrm{~g}$. As fêmeas também apresentaram o maior peso médio $(41,59 \pm 21,18 \mathrm{~g})$ em relação aos machos $(27,78 \pm 13,2 \mathrm{~g})$, assim como, tendo o maior comprimento médio $(16,5 \pm 2,8 \mathrm{~cm})$ do que os machos $(14,44 \pm 2,1 \mathrm{~cm})$. Os imaturos tiveram peso médio de $6,75 \pm 6,25 \mathrm{~g}$ e comprimento médio de $8,34 \pm 3 \mathrm{~cm}$ (Tabela 1 ).

Quando todo período das coletas foi analisado, houve diferença $\left(\chi^{2}=17,06 ; \mathrm{p}<0,05\right)$ entre as distribuições de comprimentos de machos e de fêmeas de Oligosarcus hepsetus capturados no Rio Grande. Porém, nas proporções mensais de machos e de fêmeas não 
Tabela 1. Dados biológicos referentes ao peso total capturado (PT), peso médio (PM) e comprimento médio (CM) para Oligosarcus hepsetus de machos, fêmeas, imaturos e total.

Table 1. Biological data regarding the captured total weight (WT), mean weigh (WM) and mean length (LM) of males, females, immature and total for Oligosarcus hepsetus.

\begin{tabular}{lcccc}
\hline & Total & Machos & Fêmeas & Imaturos \\
\hline PT $(\mathrm{g})$ & $3.416,98$ & $1.444,91$ & $1.830,22$ & 141,85 \\
$\mathrm{PM} \pm \mathrm{DP}(\mathrm{g})$ & $29,2 \pm 20,2$ & $27,78 \pm 13,2$ & $41,59 \pm 21,8$ & $6,75 \pm 6,25$ \\
$\mathrm{CM} \pm \mathrm{DP}(\mathrm{cm})$ & $14,12 \pm 3,8$ & $14,44 \pm 2,1$ & $16,5 \pm 2,8$ & $8,34 \pm 3$ \\
$\mathrm{~N}$ & 117 & 52 & 44 & 21 \\
\hline
\end{tabular}

Tabela 2. Representação algébrica das retas e curvas de função potência da relação peso-comprimento para os grupos de Oligosarcus hepsetus. N = número de indivíduos analisados, $\mathrm{r}=$ coeficiente de correlação linear de Pearson com sua significância.

Table 2. Algebric representation of the straight line and curves of function potency of the length-weight relationship for the groups of Oligosarcus hepsetus. $\mathrm{N}=$ number of analyzed individuals, $\mathrm{r}=$ Pearson's correlation coefficient with significance.

\begin{tabular}{lrlll}
\hline Grupo & $\mathbf{N}$ & Reta & Curva & r \\
\hline Total & 117 & $\ln y=1,0084+3,0042 \ln \mathrm{x}$ & $\mathrm{y}=0,0084 \mathrm{x}^{3,0042}$ & $0,996(\mathrm{p}<0,01)$ \\
Fêmeas & 44 & $\ln \mathrm{y}=1,0080+3,0197 \ln \mathrm{x}$ & $\mathrm{y}=0,0079 \mathrm{x}^{3,0197}$ & $0,998(\mathrm{p}<0,01)$ \\
Machos & 52 & $\ln \mathrm{y}=1,0079+3,0308 \ln \mathrm{x}$ & $\mathrm{y}=0,0078 \mathrm{x}^{3,0308}$ & $0,978(\mathrm{p}<0,01)$ \\
Imaturos & 21 & $\ln \mathrm{y}=1,0090+2,9702 \ln \mathrm{x}$ & $\mathrm{y}=0,0089 \mathrm{x}^{2,9702}$ & $0,998(\mathrm{p}<0,01)$ \\
\hline
\end{tabular}

houve diferenças $\left(\chi^{2}=8,42 ; \mathrm{gl}=11 ; \mathrm{p}>0,05\right)$, como também nas sazonais $\left(\chi^{2}=0,76 ; \mathrm{gl}=3 ; \mathrm{p}>0,05\right)$.

A Tabela 2 mostra as expressões das linhas retas estimadas após transformação logarítmicas dos dados de peso e comprimento e das curvas de função potência obtidas a partir delas.

A Figura 1 mostra a correlação entre o fator de condição $\left(\mathrm{K}_{1}\right)$ com o comprimento total de Oligosarcus hepsetus para indivíduos fêmeas, machos e imaturos, onde pode ser visto que o fator de condição não varia com o comprimento do peixe. Nota-se também que o fator de condição médio (linha horizontal na Figura 1) corresponde aos valores de a na expressão $\mathrm{y}=\mathrm{ax}^{\mathrm{b}}$, que também é o fator de condição.

Todavia, foi constatada a variação significativa do fator de condição entre os grupos de fêmeas, machos e imaturos pela prova de Kruskal-Wallis $(H=29,03$; $p<0,001)$. Pelo teste "a posteriori" os imaturos apresentaram um valor médio do fator de condição $(\mathrm{K}=0,0089)$ superior $(\mathrm{p}<0,001)$ ao de fêmeas $(\mathrm{K}=0,0079)$ e de machos $(K=0,0078)$, que foram semelhantes $(p>0,05)$.

A Figura 2 mostra a variação sazonal do fator de condição para fêmeas e machos, considerando o fator de condição calculado com o peso das gônadas $\left(K_{1}\right)$ e sem o peso da gônada $\left(K_{2}\right)$ e os respectivos intervalos de confiança. Observa-se que no outono, tanto as fêmeas como os machos apresentaram valores mais elevados, indicando uma boa condição neste período. Para as fêmeas, no inverno e na primavera, há uma maior diferença entre os fatores de condição $\mathrm{K}_{1}$ e $\mathrm{K}_{2}$ em decorrência do maior desenvolvimento dos ovários, sendo que esta diferença torna-se menor no período em que os ovários estão em repouso, no outono. Para os machos, a diferença entre $K_{1}$ e $K_{2}$ é menos conspícua nas estações do ano, pela menor contribuição do peso dos testículos no peso total do peixe. No outono, tanto para as fêmeas como para os machos, o fator de condição encontra-se elevado pelo fato de estarem em repouso, fora do período mais intenso de reprodução.

\section{Discussão}

O fator de condição é um índice que indica o estado de bem estar do peixe e que não deve variar com o comprimento do peixe, sendo correlacionado com a constante da relação peso-comprimento, expressa por $\mathrm{P}=\mathrm{aC}^{\mathrm{b}}$ (Braga 1986). Os valores individuais do fator de condição alométrico não variaram com o comprimento de peixes
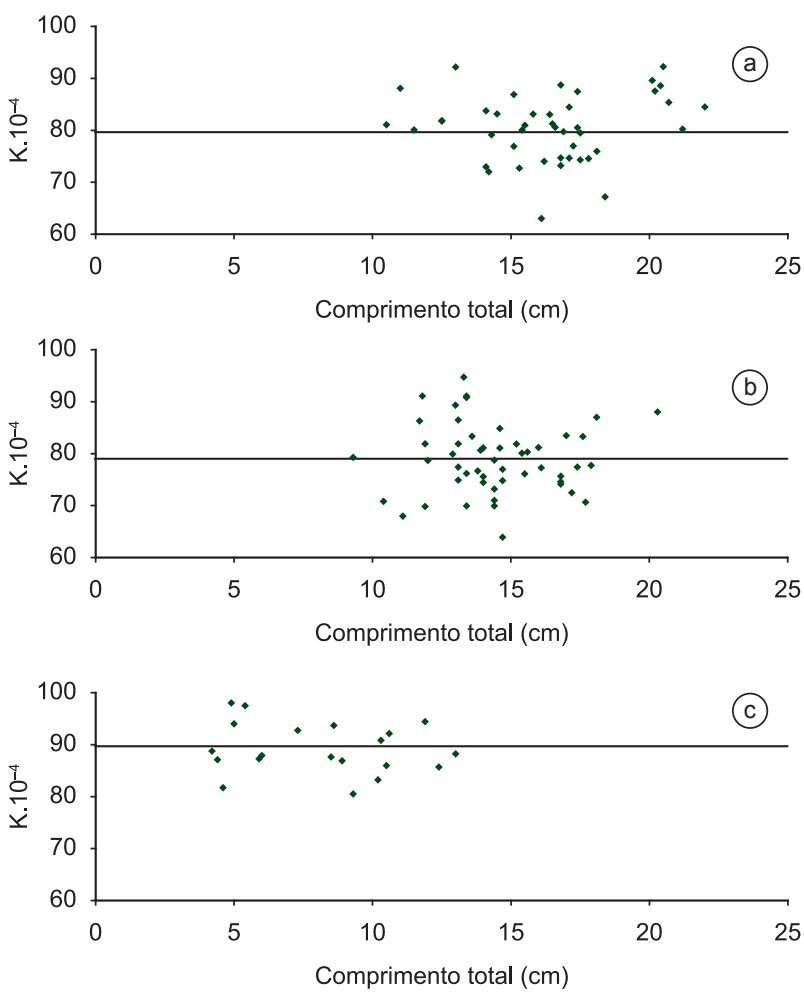

Figura 1. Relação do fator de condição com o comprimento total de fêmeas (a), machos (b) e imaturos (c) e a linha de tendência.

Figure 1. Relationship of the condition factor with the total length of females (a), males (b) and immature (c) and the tendency line.

adultos (fêmeas e machos) e nem com o comprimento de peixes imaturos. Porém, o fator de condição médio foi similar à constante a da relação peso-comprimento de fêmeas e machos $(0,0079$ e 0,0078 , respectivamente) e foi superior para os imaturos $(0,0089)$.

$\mathrm{O}$ fator de condição superior para os imaturos, sugere que neste caso a condição dos peixes, que ainda não entraram no ciclo reprodutivo e que estão em fase de crescimento rápido, direcionando os produ- 


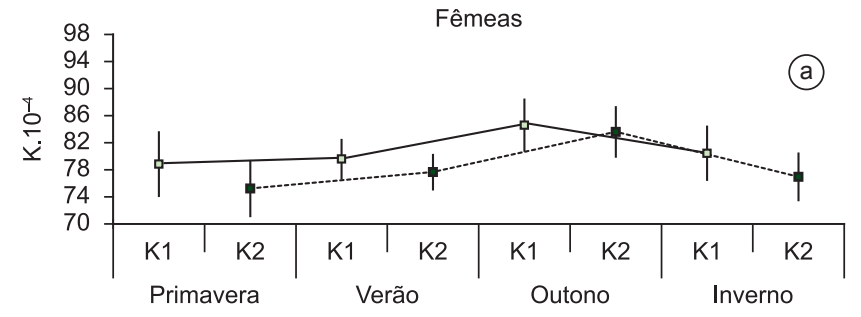

Estações do ano

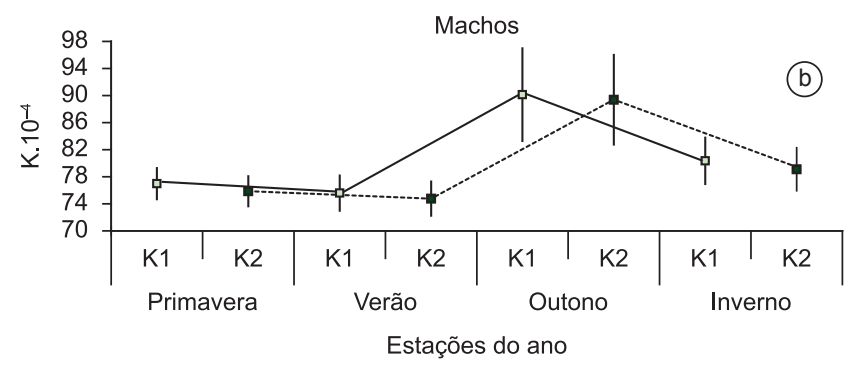

Figura 2. Variações das médias de K1 e K2 com seus respectivos intervalos de confiança para fêmeas e machos de Oligosarcus hepsetus em cada estação do ano.

Figure 2. Variations of the means of $\mathrm{K} 1$ and $\mathrm{K} 2$ with their respective confidence intervals for females and males of Oligosarcus hepsetus in each season.

tos do alimento ingerido para a construção do corpo, apresentam um valor superior para o fator de condição. Em classes de comprimentos menores, antes de atingir a primeira maturação gonadal, o peixe cresce mais linearmente do que em volume, após atingir a primeira maturação gonadal o ganho em peso torna-se proporcionalmente maior, enquanto que o crescimento linear diminui e isso é representado por uma curva de função potência (Weatherley \& Gill 1987).

Os indivíduos jovens que ainda não alcançaram o comprimento de primeira maturação gonadal frequentemente apresentam altos valores de fator de condição (Barbieri et al. 1985, Agostinho et al. 1990, Lizama \& Ambrósio 2002, Villares Jr. et al. 2007). O fator de condição para Brycon opalinus (Cuvier 1819), no mesmo ambiente de Oligosarcus hepsetus, foi menor para as fêmeas que apresentaram o comprimento de primeira maturação gonadal $\left(\mathrm{L}_{50}\right)$ e foi maior para os indivíduos imaturos (Gomiero \& Braga 2006). O fator de condição pode ser influenciado pelo estádio de maturação das gônadas e pelo grau de repleção dos estômagos ao longo do tempo Barbieri et al. (1982, 1985).

Nos ecossistemas aquáticos a sazonalidade influencia os períodos reprodutivos e a frequência alimentar das espécies de peixes (Vazzoler 1996). Em ambientes com pouca variação sazonal como a Mata Atlântica, estes eventos podem se estender por mais tempo, sendo que apenas parte da população de uma espécie reproduz ao mesmo tempo e a alimentação e acúmulo de gordura podem acontecer na maior parte do ano. Sobre isto, Gomiero et al. (2008) verificaram que as distribuições das frequências dos estádios de maturidade gonadal foram sazonalmente diferentes para machos e fêmeas de Oligosarcus hepsetus com longo período reprodutivo de 8 meses. Os valores de comprimento médio de primeira maturação gonadal $\left(\mathrm{L}_{50}\right)$ para machos e fêmeas foi de 11,2 e 11,6 cm, respectivamente, e o comprimento em que todos os indivíduos já são adultos $\left(\mathrm{L}_{100}\right)$ foi de $16 \mathrm{~cm}$ para ambos os sexos. Já os imaturos foram frequentes em todas as estações do ano, com maior número de indivíduos no fim do outono e no inverno. Apresentando assim, longo período reprodutivo, comprimento de primeira maturação gonadal precoce e desova parcelada, sendo considerado típico estrategista oportunista, segundo Winemiller (1989).

As variações sazonais da diferença entre $K_{1}$ e $K_{2}$ são influenciadas pela reprodução das espécies (Vazzoler 1996, Gomiero \& Braga 2006). Como para Brycon opalinus (Gomiero \& Braga 2006) e Rhamdia quelen (Quoy \& Gaimard 1824) (Gomiero et al. 2007) no mesmo ambiente, $O$. hepsetus apresentou longo período reprodutivo evidenciado pelas diferenças do $K_{1}$ e $K_{2}$ no inverno, na primavera e no verão.

Os maiores valores do fator de condição de $O$. hepsetus no outono possivelmente ocorreu devido a maior intensidade alimentar no final do inverno e na primavera, com subsequente acúmulo de gordura no final do verão e principalmente no outono (Botelho et al. 2007). Por outro lado, os valores inferiores do fator de condição no inverno, na primavera e no verão, coincidem com o período reprodutivo e respectivo aumento do gasto energético para a produção dos gametas.

A plasticidade fisiológica e comportamental dos peixes ajusta-se às inúmeras diferenças ambientais encontradas nas áreas de ocorrência dessas espécies. A sobrevivência das populações em áreas diferentes depende desta adaptabilidade ambiental influenciando os eventos reprodutivos e alimentares ao longo do ciclo de vida.

As espécies de peixes analisadas nos rios do Parque Estadual da Serra do Mar - Núcleo Santa Virgínia convergem para estratégias muito próximas quanto ao longo período reprodutivo, desova parcelada, baixa fecundidade, comprimento de primeira maturação gonadal precoce e longo período alimentar. A baixa variação ambiental ao longo dos períodos, a disponibilidade constante de recursos e as condições encontradas na Mata Atlântica parecem ser primordiais para o desenvolvimento das estratégias apresentadas pela ictiofauna.

\section{Conclusão}

As fêmeas e os machos tiveram fator de condição mais alto no outono (época em que ocorreu o maior acúmulo de gordura). $\mathrm{O}$ fator de condição dos imaturos foi mais alto do que para machos e fêmeas, possivelmente devido ao gasto energético com o desenvolvimento dos ovócitos e em menor grau dos espermatozóides. A área de estudo encontra-se na Mata Atlântica onde existe pouca variação sazonal nas condições ambientais e disponibilidade constante de recursos ao longo do ano, fazendo com que Oligosarcus hepsetus apresentasse longo período reprodutivo evidenciado pelo fator de condição $\mathrm{K}_{1}$ e $\mathrm{K}_{2}$ de fêmeas e machos no inverno, na primavera e no verão. As estratégias apresentadas pela ictiofauna relacionam-se com as condições ambientais prevalecentes ao longo de todo o ano para a alimentação e reprodução, condições essas necessárias para a manutenção e abundância das espécies.

\section{Referências}

AGOSTINHO, A.A., BARBIERI, G., VERANI, J.R. \& HANH, N.S. 1990. Variação do fator de condição e do índice hepatossomático e suas relações com o ciclo reprodutivo em Rhinelepis aspera (Agassis, 1829) (Osteichthyes, Loricariidae) no rio Paranapanema, Porecatu, PR. Ciênc. Cult. 42(9):711-714.

ARAÚJO, F.G. 1998. Adaptação do índice de integridade biótica usando a comunidade de peixes para o rio Paraíba do Sul. Braz. J. Biol. 58(4):547-558.

ARAÚJO, F.G., ANDRADE, C.C., SANTOS, R.N., SANTOS, A.F.G.N. \& SANTOS, L.N. 2005. Spatial and seasonal changes in the diet of Oligosarcus hepsetus (Characiformes, Characidae) in a brazilian reservoir. Rev. Bras. Zool. 65(1):1-8.

ARAÚJO-LIMA, C.A.R.M., HIGUCHI, N. \& BARRELLA, W. 2004. Fishesforestry interactions in tropical South América. In Fishes and forestry (T.G. Northcote \& G.F. Hartman, eds.). Blackwell Science, Vancouver, p. 511-534. 
BARBIERI, G., SANTOS, M.V.R. \& SANTOS, J.M. 1982. Época de reprodução e relação peso/comprimento de duas espécies de Astyanax (Pisces, Characidae). Pesq. Agrop. Bras. 17(7):1057-1065.

BARBIERI, G., VERANI, J.R., PEREIRA, J.A., BARBIERI, M.C., PERET, A.C. \& MARINS, M.A. 1985. Curva de maturação e fator de condição de Apareiodon affinis (Steindachner, 1879), Apareiodon ibitiensis (Campos, 1944) e Parodon tortuosos (Eigenman \& Norris, 1900) do rio Passa Cinco Ipeúna-SP (Cypriniformes, Parodontidae). Ciênc. Cult. 37(7):1178-1183.

BENEDITO-CECILIO, E., MINTE-VERA, C.V., ZAWADZKI, C.H., PAVANELLI, C.S., RODRIGUES, F.H.G. \& GIMENES, M.F. 2004. Ichthyofauna from the Emas National Park region: composition and structure. Braz. J. Biol. 64(3):371-382.

BOTELHO, M.L.L.A., GOMIERO, L.M. \& BRAGA, F.M.S. 2007. Feeding of Oligosarcus hepsetus (Cuvier, 1829) (Characiformes) in the Serra do Mar State Park - Santa Virgínia Unit, São Paulo, Brazil. Braz. J. Biol. 67(4):741-748

BRAGA, F.M.S. 1986. Estudo entre fator de condição e relação peso-comprimento para alguns peixes marinhos. Braz. J. Biol. 46(2):339-346.

BRAGA, F.M.S. 1993. Análise do fator de condição de Paralonchurus brasiliensis (Perciformes, Sciaenidae). Rev. UNIMAR 15(2):99-115.

BRAGA, F.M.S. 1997. Análise da equação alométrica na relação peso e comprimento e o fator de condição em Plagioscion squamosissimus (Teleostei, Sciaenidae). Braz. J. Biol. 57(3):417-425.

BRAGA, F.M.S. \& GENNARI FILHO, O. 1990. Contribuição para o conhecimento da reprodução de Moenkhausia intermedia (Characidae, Tetragonopterinae) na represa de Barra Bonita, rio Piracicaba, SP. Naturalia 15:171-188.

BRAGA, F.M.S., BRAGA, M.A.S. \& GOITEIN, R. 1985. Fator de condição e alimentação de Paralonchurus brasiliensis (Osteichthyes, Sciaenidae) na região da ilha Anchieta (lat. $23^{\circ} 33^{\prime} \mathrm{S}$ - long. $45^{\circ} 05^{\prime} \mathrm{W}$ ) Ubatuba, Estado de São Paulo. Naturalia 10:1-11.

CASTRO, R.M.C. \& MENEZES, N.A. 2001. Estudo diagnóstico da diversidade de peixes do Estado de São Paulo. http://www.biota.org. br/iLink?livros.biota+Peixes+volseis+ Diagnostico (último acesso em 17/09/2001).

GOMIERO, L.M. \& BRAGA, F.M.S. 2003. Relação peso-comprimento e fator de condição para Cichla cf. ocellaris e Cichla monoculus (Perciformes, Cichlidae) no reservatório de Volta Grande, rio Grande - MG/SP. Acta Sci. 25(1):79-86

GOMIERO, L.M. \& BRAGA, F.M.S. 2005. The condition factor of fishes from two river basins in São Paulo State, Southeast of Brazil. Acta Sci. 27(1):73-78.

GOMIERO, L.M. \& BRAGA, F.M.S. 2006. Relação peso-comprimento e fator de condição de Brycon opalinus (Pisces, Characiformes) no Parque Estadual da Serra do Mar - Núcleo Santa Virgínia, Mata Atlântica, Estado de São Paulo, Brasil. Acta Sci. 28 (2):135-141.

GOMIERO, L.M., SOUZA, U.P. \& BRAGA, F.M.S. 2007. Reprodução e alimentação de Rhamdia quelen (Quoy \& Gaimard, 1824) em rios do Núcleo Santa Virgínia, Parque Estadual da Serra do Mar, São Paulo, SP. Biota Neotrop. 7(3):1-7.

GOMIERO, L.M., GARUANA, L. \& BRAGA, F.M.S. 2008. Reproduction of Oligosarcus hepsetus (Cuvier, 1829)(Characiforms) in the Serra do Mar State Park, São Paulo, Brazil. Braz. J. Biol. 68(1):195-200.
LE-CREN, E.D. 1951. The length-weight relationship and seasonal cycle in gonad weight and condition in the perch (Perca fluviatilis). J. Anim. Ecol. 20(2):201-219.

LIZAMA, M.A.P. \& AMBRÓSIO, A.M. 2002. Condition factor in nine species of fish of the Characidae family in the upper Paraná river floodplain, Brazil. Braz. J. Biol. 62 (1):113-124.

LOTRICH, V.A. 1973. Growth, production, and community composition of fishes inhabiting a first-second, and third-order stream of eastern Kentucky. Ecol. Monogr. 43(3):377-397.

MENEZES, N.A. 1987. Três espécies novas de Oligosarcus Gunther, 1864 e redefinição taxonômica das demais espécies do gênero (Osteichthyes, Teleostei, Characidae). Bolm. Zool. 11:1-39.

MENEZES, N.A. 2003. Padrões de distribuição da biodiversidade da Mata Atlântica do sul e sudeste brasileiro: peixes de água doce. http://www. bdt.fat.org.br/workshop/mata.atlantica/SE-S/peixes (último acesso em 18/03/2003).

NIKOLSKII, G.V. 1963. The ecology of fishes. Academic Press, London, $352 \mathrm{p}$.

NOMURA, H. 1962. Length-weight tables of some fish species from southern Brazil. Instituto Oceanográfico da Universidade de São Paulo, São Paulo 2:1-84

POPOVA, O.A. 1978. The role of predaceous fish in ecosystems. In Ecology of freshwater fish production (S.D. Gerking, ed.). Blackwell Scientific, Oxford, p. 215-249.

RICKER, W.E. 1975. Computation and interpretation of biological statistics of fish populations. Bull. Fish Res. Board. Can. 191:1-382.

SANTOS, E.P. 1978. Dinâmica de populações aplicada à pesca e piscicultura. Hucitec, São Paulo, 129 p.

TONHASCA Jr, A. 2005. Ecologia e história natural da Mata Atlântica. Editora Interciência, Rio de Janeiro, 197 p.

VANZOLINI, P.E. 1993. Métodos estatísticos elementares em sistemática zoológica. Hucitec, São Paulo, 130 p.

VAZZOLER, A.E.A.M. 1996. Biologia da reprodução de peixes teleósteos: teoria e prática. Nupélia, Maringá, 169 p.

VILLARES Jr, G.A., GOMIERO, L.M. \& GOITEIN, R. 2007. Relação peso-comprimento e fator de condição de Salminus hilarii Valenciennes 1850 (Osteichthyes, Characidae) em um trecho da bacia do rio Sorocaba, Estado de São Paulo, Brasil. Acta Sci. 29(4):407-412.

WEATHERLEY, A.H. 1972. Growth and ecology of fish populations. Academic Press, London, 293 p.

WEATHERLEY, A.W. \& GILL, H.S. 1987. The biology of fish growth Academic Press, London, 443 p.

WINEMILLER, K. 1989. Patterns of variation in life history among South American fishes in seasonal environments. Oecologia 81(2):225-241.

ZAR, J.H. 1996. Biostatistical analysis. 3 ed. Prentice-Hall International Editions, New Jersey, 718 p.

Recebido em 30/05/09 Versão reformulada recebida em 08/12/09 Publicado em 12/02/10 